\title{
An economic model to estimate the relative costs over 20 years of different hip prostheses
}

\author{
Kate Baxter, Gwyn Bevan
}

\begin{abstract}
Study objective-To determine the relative costs of prostheses and factors associated with changes in these cost rankings. Design and setting-Economic model using published data.

Main results-The main cost drivers are current costs and revision rates. Expected revision costs are a small proportion of the expected total costs. There are few competitors to the "gold standard" Charnley in terms of total expected costs over 20 years. There is no monetary advantage in using higher cost prostheses in older patients even if they were to have lower revision rates. There may be a monetary case for using prostheses with higher costs and lower revision rates in younger patients.

Conclusions-The most cost effective prosthesis in older patients is the Stanmore. The Charnley, Exeter Polished and Muller Straight Stem are marginally more costly than the Stanmore. The study inevitably lacks good data on survival for newer prostheses. This does not affect the ability to make choices for older patients. Data are needed, however, on survival of cemented prostheses for younger patients. (F Epidemiol Community Health 1999;53:542-547)
\end{abstract}

Total hip replacement has, since the 1960s, become one of the most frequently undertaken orthopaedic procedures in the National Health Service: there were 32500 primary replacements undertaken in 1994/5. ${ }^{1}$ Total hip replacements are extremely effective in pain relief and improved physical function. The technology of hip prostheses is continually changing, with many new designs and methods of fixation. As total hip replacements have been extended to younger age groups, an increase in the longevity of implants is being sought. There is no statutory or nationally coordinated monitoring of processes of innovation and diffusion in the United Kingdom. Orthopaedic innovation in total hip replacement technology internationally has been referred to as a "trial and error culture" and there are over 60 different models of prosthesis used in the UK. There is a rising number of prostheses available, with little or no scientific evidence that the newer implants are any better than the established ones. ${ }^{3}$ The warning in February 1998 from the Medical Devices Agency ${ }^{4}$ about the unacceptably high failure rate of the $3 \mathrm{M}$ Capital hip system, combined with the fact that 4669 of these untested hips have been used in 95 centres throughout Britain, highlights this lack of regulation. ${ }^{5}$
This paper describes the relative cost effectiveness of total hip replacements, based on total expected costs over 20 years, using a selection of hip prostheses available in the UK. The viewpoint taken is that of a purchaser.

We use a modified version of the model developed in papers by Daellenbach et $a l^{6}$ and Gillespie et $a .^{7}$ Both these papers give the conditions necessary for cementless prostheses to be of equal or greater cost effectiveness than cemented prostheses. Both also stated concerns about the lack of adequate data on failure rates of established and newly developed prostheses. Extensive sensitivity analysis was carried out by Daellenbach et al $l^{6}$ on the cost parameters of the model where their accuracy was uncertain.

Pynsent $e t a l^{8}$ introduced a new concept of "lifetime care packages". With such a package, purchasers, rather than paying for subsequent revisions, would pay an insurance premium to providers at the time of the primary replacement, to cover the expected costs of revision.

Our paper adds to this previous work by using up to date hospital and prosthesis costs to give cost rankings for 15 prostheses. We also test the importance of each component of the model in estimating total expected costs and give simple graphs for comparison of total expected costs for hypothetical ranges of prosthesis prices and revision rates. The model can use new and local data as these become available.

\section{Methods}

COST MODEL

A model was developed to give the present value of using each prosthesis. The present value of prosthesis $j$ is dependent upon three components: the costs of the initial prosthesis; hospital costs of the primary procedure; and the sum of expected future costs of revision. Future costs of revision depend on the age of the recipient and the survival of the implant. All future costs are discounted. The model is based on that used by Daellenbach et $a l^{6}$ and Gillespie et $a l^{7}$ and is of the form

$\mathrm{PV}_{j}=\mathrm{C}_{\mathrm{j}}+\mathrm{H}+\sum\left\{\frac{\mathrm{Lmi} \cdot \mathrm{P} j m i \cdot\left(\mathrm{C}_{\mathrm{j}}+\mathrm{H}+\mathrm{R}\right)}{(1+\mathrm{r})^{\mathrm{i}}}\right\}$

where

- $\mathrm{PV} j=$ present value of using prosthesis $\mathrm{j}$

- $\mathrm{C}_{\mathrm{j}}=$ cost of prosthesis $\mathrm{j}$

- $\mathrm{H}=$ hospital costs including separate categories for

- theatre costs

- ward costs

- prophylaxis costs

- physiotherapy costs 
- L $m i=$ probability of a person at age $m$ when receiving a hip replacement being alive in year $i$

- $\operatorname{Pjmi}=$ probability of prosthesis $j$ in person aged $m$ needing to be revised in year $i$

- $\mathrm{R}=$ additional costs of a revision - that is, additional hospital costs

- $1 /(1+r)^{\mathrm{i}}=\mathrm{a}$ discount factor where $\mathrm{r}$ is the discount rate, $i=0$ to 19 where 0 is the year of the primary operation

\section{SENSITIVITY ANALYSIS}

Sensitivity analysis is a method of dealing systematically with uncertainties in economic evaluations. It tests the robustness of the conclusions with respect to the data used. Simple sensitivity analysis is the most common, and entails changing one or more variables. One way analysis entails changing each variable individually to determine the effect on the final result of each variable. Multi-way analysis entails changing more than one variable, and can be used to consider different scenarios or "states of the world". 9

The effect on total expected costs of each variable in the equation was tested using one way analysis. Total expected costs using a lowest and a highest possible value for each variable were compared with costs using the best estimate. The results indicate which variables have the greatest effect on total expected costs. The maximum and minimum values used in the sensitivity analysis are taken from either published data or data provided by the collaborating hospitals.

DATA SOURCES

Data used in the model were provided by two sources: two collaborating hospitals in different regions of England and published work.

The collaborating hospitals provided the average price they charged to purchasers for a hip prosthesis $\left(C_{j}\right)$ in 1996/7. This allowed actual costs in the two hospitals to be modelled. Prosthesis prices quoted in Murray et al $l^{\beta}$ were also used to enable the model to compare expected costs of differing prostheses. Murray et $a l^{\beta}$ gives 1994 prices. These have been inflated using annual percentage price increases in hospital and community health services ${ }^{10}$ to provide data in the same base year as the hospital costs (1996/7). The well established and relatively cheap Charnley prosthesis has been used as the "gold standard" for the basic modelling and comparison against other prostheses. The range of market prices given by Murray has been used in the sensitivity analysis.

Hospital costs $(\mathrm{H})$ were given by the collaborating hospitals, broken down into theatre, ward, physiotherapy, and prophylaxis costs. The effects of long and short theatre times and lengths of stay were examined through sensitivity analysis.

Costs of revision surgery are based on one hospital's estimated additional costs of revision (R) as 1.19 times the price of a primary unilateral replacement (excluding prosthesis). These additional costs of revision were made proportional to the primary replacement costs in the model to allow estimates used in the
KEY POINTS

- Changes in prosthesis cost, hospital costs, and revision rates are the main drivers of total expected costs of hip replacements.

- For recipients aged 70 years old, the expected costs of revisions over 20 years are small.

- For younger recipients, relatively expensive prostheses with low revision rates can be less costly over 20 years than cheaper prostheses with higher revision rates.

- The commonly used and relatively cheap prostheses are also the least costly prostheses over 20 years.

sensitivity analyses to impact on both primary and revision costs.

The probability of a person at age $m$ when receiving a primary hip replacement being alive in year $i(\mathrm{Lm} i)$ was calculated from OPCS Mortality Statistics (1992). ${ }^{11}$ The probabilities for men and women were modelled separately in the equation. Median age at primary operation (70 years) was calculated from data supplied by one of the hospitals. In the absence of any other data we have assumed that people who have had a hip replacement have a mortality rate equal to the general population for their age group. This, however, is unlikely and its implications are considered in the Discussion.

The probability of prosthesis $j$ needing revision in year $i$ (P $j m i)$ was estimated from published sources. ${ }^{3}{ }^{12-16}$ A number of survival rates ranging from survival at one year to survival at 20 years were available for the Charnley. More limited data were available for other prostheses. The data from these sources were collated and a best estimate of the probability of revision in each year over 20 years was calculated. Gaps in the data were filled assuming a straight line relation between two known points. Total expected costs for assumed straight line revision rates over 20 years of $0 \%$ to $5 \%$ per year were also modelled.

The discount factor applied to a cost 20 years into the future, using a discount rate of $5 \%$, is around 0.39 : that is, the present value of a cost incurred in 20 years time is only about $40 \%$ of its nominal value. This model estimates the total expected costs over a maximum of 20 years. For consistency with many published evaluations, the discount rate chosen for the main calculation was $5 \% .{ }^{17}$ This figure was varied in the sensitivity analysis to $0 \%$ and $6 \%$ (the current Treasury discount rate).

\section{ASSUMPTIONS AND EXCLUSIONS}

This model is based on a number of simplifying assumptions:

(1) Prosthesis revision rates are linear where long term survival data are not known.

(2) Mortality rates of total hip replacement recipients are equal to those of the general population.

(3) Account is taken in the model of first revisions but not re-revisions. 
Table 1 Present (cumulative) value of expected revision costs for men and woman for a selection of years (average hospital prosthesis and Charnley prosthesis costs separately)

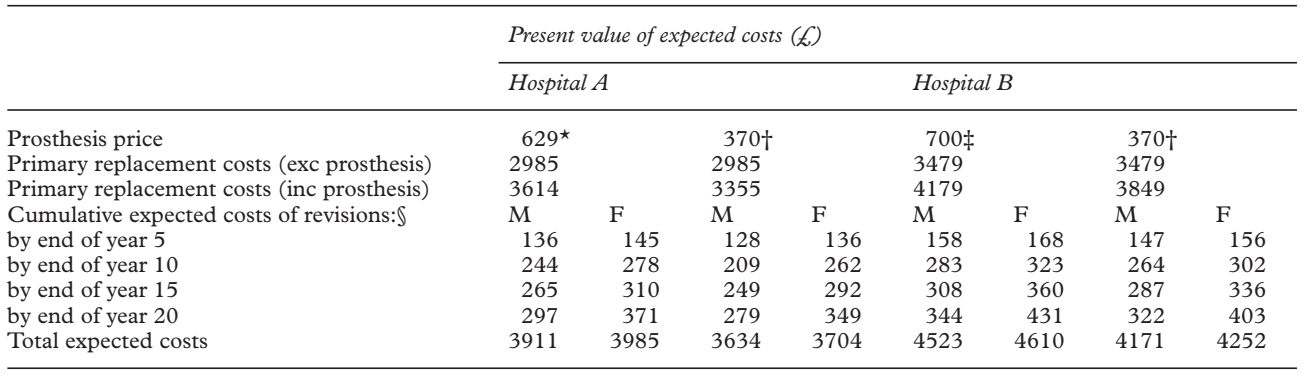

*Average prosthesis cost in hospital A, including cement (71). †Cost of Charnley prosthesis from Murray et al plus 71 cement. $\ddagger$ Average prosthesis cost in hospital B. \Assuming revision rates of the Charnley prosthesis.

(4) Hospital costs are the same for all types of prosthesis and all patients.

Costs to the patient and their families are not included, nor are costs of rehabilitation in the community. Benefits to the patient, other than implicitly in the total hip replacement revision rate, are not included. Total hip replacement is known to be clinically effective, the purpose of this model is to compare long term costs of alternative prostheses, not to justify total hip replacement itself.

USES OF THE MODEL

By modelling the total expected costs of total hip replacements, comparisons between different prostheses can be made. For example, assume a choice between two prostheses: Y needing no revisions over 20 years, and $\mathrm{X}$ having, say, a $1 \%$ per annum revision rate. Prosthesis Y costs $£ 1000$ more than prosthesis $\mathrm{X}$. A purchaser, making a decision based on expected costs only, would chose $Y$ rather than $\mathrm{X}$ if the expected costs of revisions of prosthesis $\mathrm{X}$ over the next 20 years were more than $£ 1000$ (and vice versa).

The method can also be used for making comparisons where costs are known but survival data are not. For example, assume for a new prosthesis $\mathrm{A}$, its cost is known but no survival data are available. Both costs and survival data are known for an established prosthesis $\mathrm{B}$. If the primary replacement costs of prosthesis A are greater than total expected costs of prosthesis $\mathrm{B}$, then it is inevitable that the total expected costs of prosthesis A will be greater than those of prosthesis B.

\section{Results}

Table 1 gives the present value of expected revision costs over 20 years, separately for men and women aged 70 at replacement. Charnley revision rates calculated from published sources (range $0.5 \%$ and $3 \%$ revisions per year) are used. Two prosthesis prices are used for each hospital: the prosthesis prices charged by the respective hospitals, and the cost of a Charnley from published data. ${ }^{3}$ The prosthesis prices quoted by the hospitals are average prices of prostheses. The mix of prosthesis types that these averages represent is not known. However, from a purchasers point of view, the charge is part of the standard price of a total hip replacement. These hospital prosthesis prices are higher than the price of a Charnley given in Murray et al. ${ }^{3}$ Results using both prices are therefore included to show the actual as well as the possible costs. Primary replacement costs, the present value of cumulative expected costs of revisions (shown at five year intervals), and total expected costs are shown. Expected revision costs over 20 years for women are slightly higher than for men.

For male patients in Hospital A, using the hospital's own data, the expected cost of revisions over 20 years is £297. The total expected costs are therefore the primary replacement costs plus the $£ 297$. If a hypothetical new prosthesis needed no revisions over 20 years, its expected cost would be equal to its primary replacement costs. A purchaser would therefore be prepared to buy this "no revisions" prosthesis if its cost was no more than $£ 297$ greater than the current prosthesis. Paying more than $£ 297$ extra (that is, $£ 926$ in total) for the "no revisions" prosthesis would result in the costs over 20 years being greater than the costs using the current implant. Using data for Hospital B, the maximum extra a purchaser would be prepared to pay for a "no revision" prosthesis is slightly higher at $£ 344$ ( $£ 973$ total).

For simplicity, the equation used in the sensitivity analysis and the comparison of different

Table 2 Sensitivity analysis: effects on total expected costs of modelling minimum and maximum estimates of costs (women)

\begin{tabular}{|c|c|c|c|c|c|c|}
\hline & Best estimate* $(f)$ & Low estimate $(£)$ & High estimate $(f)$ & $\begin{array}{l}\text { Total expected cost } \\
\text { using low estimate } \\
(£)\end{array}$ & $\begin{array}{l}\text { Total expected cost } \\
\text { using high estimate } \\
\text { (£) }\end{array}$ & $\begin{array}{l}\text { Difference in } \\
\text { total expected } \\
\text { cost }(£)\end{array}$ \\
\hline Prosthesis & 370 & $336+$ & $1336 \dagger$ & 3667 & 4753 & 1086 \\
\hline Time in theatre & 144 mins & 60 mins $\ddagger$ & 246 mins $\ddagger$ & 2932 & 4642 & 1710 \\
\hline Days on ward & 13.2 days & 9 days $\S$ & 22 days & 3096 & 4978 & 1882 \\
\hline Discount rate & $5 \%$ & $6 \%$ & $0 \%$ & 3680 & 3881 & 201 \\
\hline Additional revision costs & $19 \%$ & $0 \% \|$ & $100 \% \|$ & 3644 & 3902 & 258 \\
\hline Recipient age & 70 years & 80 years & 40 years & 3568 & 3876 & 310 \\
\hline Annual revision rate & variable $0.5 \%$ to $3 \%$ & $1 \%$ & $5 \%$ & 3722 & 5191 & 1469 \\
\hline
\end{tabular}

${ }^{\star}$ Best estimates from hospital A's costs and Charnley prosthesis price and revision rate. $†$ Low estimate from Murray $e t$ al ${ }^{3}$ high estimate $=10 w$ estimate plus 1000 . $\ddagger$ Data from hospital A. $\$$ Data from Trent Region 1990. $\|$ Data from Pynsent $e t$ al. ${ }^{8}$ Best estimate based on actual Charnley revision rates, which vary from year to year, high and low estimates based on $1 \%$ and $5 \%$ per annum revision rates. 
Table 3 Relative cost effectiveness of the Charnley and 15 comparison prostheses - best, low, and high estimates (women)

\begin{tabular}{|c|c|c|c|c|c|}
\hline \multirow[b]{2}{*}{ Make/type } & \multirow[b]{2}{*}{$\begin{array}{l}\text { Prosthesis } \\
\text { pricef }\end{array}$} & \multicolumn{4}{|c|}{ Expected costs over 20 years } \\
\hline & & $\begin{array}{l}\text { Prosthesis } \\
\text { survival at } \\
20 \text { years } \\
(\%) \ddagger\end{array}$ & $\begin{array}{l}\text { Best } \\
\text { estimateS }\end{array}$ & $\begin{array}{l}\text { Low } \\
\text { estimate }\end{array}$ & $\begin{array}{l}\text { High } \\
\text { estimate }\end{array}$ \\
\hline Charnley* & 370 & 78 & 3704 & 2323 & 5916 \\
\hline \multicolumn{6}{|l|}{ Cemented } \\
\hline Stanmore $\star \star$ & 373 & 88 & 3578 & 2247 & 5712 \\
\hline 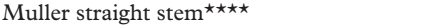 & 424 & 80 & 3696 & 2341 & 5869 \\
\hline Exeter Polished $\star \star$ & 431 & 82 & 3718 & 2357 & 5899 \\
\hline Cemented alumina-alumina (age $<50)^{\star \star \star}$ & 516 & 89 & 3780 & 2429 & 5943 \\
\hline Howse $\star \star$ & 516 & 70 & 3976 & 2554 & 6255 \\
\hline McKee-Farrar* & 516 & 49 & 4061 & 2607 & 6389 \\
\hline Cemented alumina-alumina (age $>50)^{\star \star \star}$ & 516 & 61 & 4190 & 2689 & 9595 \\
\hline Lubinous IP $P^{\star \star \star \star}$ & 516 & 88 & 3741 & 2405 & 5882 \\
\hline $\mathrm{CAD}^{\star \star \star \star}$ & 516 & 86 & 3767 & 2422 & 5924 \\
\hline Lubinous SP $P^{\star \star \star \star \star}$ & 808 & 93 & 3946 & 2645 & 6029 \\
\hline 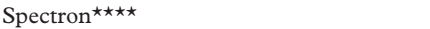 & 808 & 91 & 3971 & 2662 & 6068 \\
\hline \multicolumn{6}{|l|}{ Cementless } \\
\hline $\mathrm{AML}^{\star \star \star}$ & 837 & 75 & 4665 & 3123 & 7110 \\
\hline Omnifit ${ }^{\star \star \star \star}$ & 1320 & 97 & 4382 & 3111 & 6420 \\
\hline Harris-Galante ${ }^{\star \star \star \star \star}$ & 1205 & 87 & 4482 & 3142 & 6630 \\
\hline $\mathrm{PCA}^{\star \star \star \star}$ & 1205 & 86 & 4506 & 3158 & 6665 \\
\hline
\end{tabular}

tAll prosthesis prices from Murray et al. ${ }^{3}$ Where specific prices not quoted, average price of cemented (516) or cementless (1205) prostheses used as appropriate. $¥ 20$ year survival rates estimated from published sources. §Best estimates from hospital A’s costs and Charnley prosthesis price and revision rate. 9 Low estimate (theatre $60 \mathrm{mins}$ and ward 9 days), high estimate (theatre 246 mins and ward 22 days) (from table 2).

${ }^{\star}$ Revision data available for 20 years. ${ }^{\star \star}$ Revision data available for $15-19$ years, then extrapolated. ${ }^{\star \star \star}$ Revision data available for 10-14 years, then extrapolated. ${ }^{\star \star \star \star}$ Revision data available for 5-9 years, then extrapolated. ${ }^{\star \star \star \star \star}$ Revision data available for $<5$ years, then extrapolated.

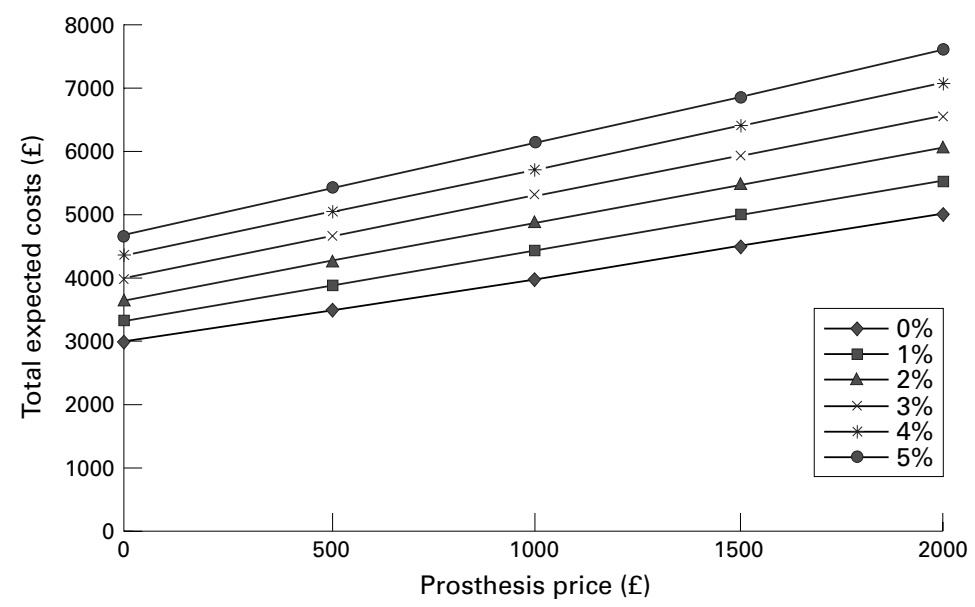

Figure 1 Relative costs over 20 years for a range of prosthesis prices and revision rates for female recipients aged 70 years (discount rate $5 \%$ ).

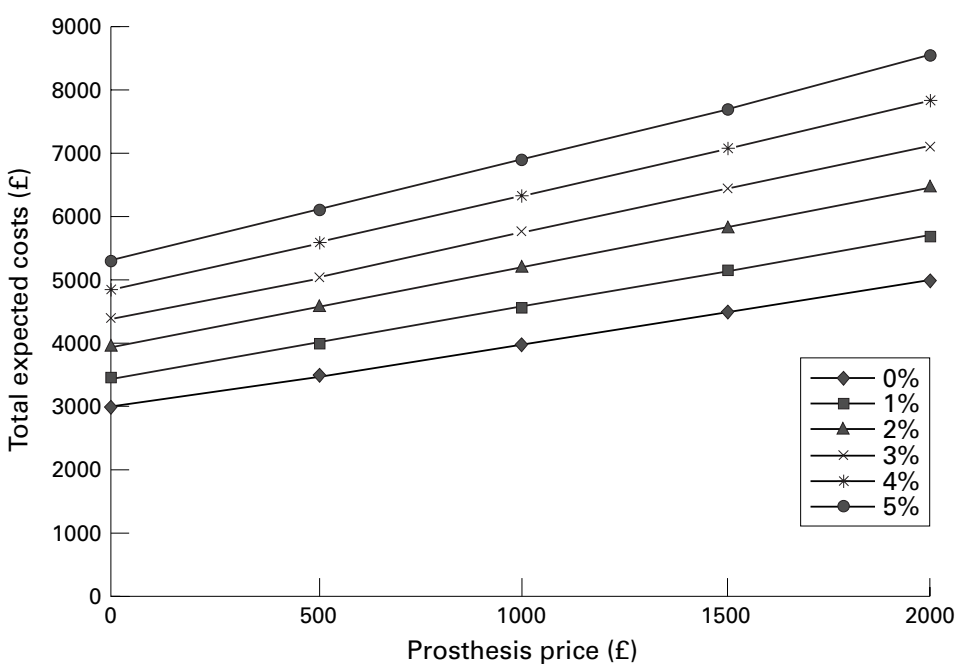

Figure 2 Relative costs over 20 years for a range of prosthesis prices and revision rates for female recipients aged 40 years (discount rate $5 \%$ ). prostheses models costs from only one hospital (Hospital A) with Charnley survival rates and Charnley prosthesis price.

Table 2 gives the results of the sensitivity analysis that examined, independently, the impact on expected costs of each of the following: prosthesis price, time in theatre, length of stay on ward, discount rate, revision costs, recipient age, and revision rate. The main cost drivers - that is, those which have the biggest impact on total expected costs-are shown to be hospital (theatre and ward) costs, price of prosthesis, and revision rate.

Table 3 models the relative costs of a range of competing prostheses with the Charnley as the "gold standard". The minimum and maximum estimates of hospital costs used in the sensitivity analysis were modelled to show any impact on cost rankings. Prostheses are classified by the number of years survival data available. Where 20 year actual survival data are available, this is indicated by a single asterisk $(\star)$, data for less than five years is shown by five asterisks $(\star \star \star \star \star)$. Survival data for all prostheses were extrapolated to 20 years. The effect of extrapolating limited prosthesis survival data is considered in the discussion. Where prosthesis prices were unknown, these were assumed to be equal to the average price paid by Hospital A. The Stanmore prosthesis appears to be marginally less costly over 20 years than the Charnley, the Exeter Polished and Muller Straight Stem, which are of similar expected costs. All four of these prostheses have prices of about $£ 400$. All the cementless prostheses have expected costs over 20 years of around $£ 700$ more than a Charnley and all are more costly than any of the cemented prostheses.

Figures 1 and 2 show expected total costs over 20 years for combinations of differently priced prostheses and revision rates for 70 and 40 year old female recipients respectively. 
Hospital costs are assumed to be the same throughout. The figures show whether greater costs of prostheses can be rewarded by lower total expected costs as a result of lower expected revision rates. For example, a $£ 1500$ prosthesis with a $1 \%$ per year revision rate has similar total expected costs over 20 years in a 40 year old as a $£ 1000$ prosthesis with a $2 \%$ revision rate, and is less costly than a $£ 500$ prosthesis with a $4 \%$ revision rate.

\section{Discussion}

This research has modelled the long term costs of different prostheses. There are currently over 60 different types of prosthesis used in the United Kingdom, ${ }^{1}$ with significant variation in price. Although reasons for consultant choice may include knowledge of prosthesis survival and function in situ, interviews with surgeons indicate that manufacturers exert a degree of influence over surgeons' preferences, with some orthopaedic departments being supplied by a single manufacturer. ${ }^{1}$ This paper makes explicit the costs associated with a range of prosthesis prices and performance.

We present the main conclusions based on our stated assumptions. We then examine how these conclusions might alter with changes in these assumptions. Finally, we discuss our findings in relation to previous work and suggest an area for future research.

Our main conclusions are:

- Compared with Charnley survival data from "centres of excellence" and prosthesis cost of $£ 370$ including cement, a "no revisions" prosthesis should cost not more than about $£ 700$ to have equivalent total expected costs over 20 years. (This result is in agreement with Daellenbach et al, ${ }^{6}$ who showed that the price of prostheses with low failure rates can be only marginally higher than those with higher failure rates for the present value of expected future costs to be comparable.)

- Prosthesis cost, hospital costs and the revision rate are the main cost drivers of total expected costs.

- Given the data used in the model, the Stanmore prosthesis appears to be less costly over 20 years than the Charnley; the Exeter Polished and Muller Straight Stem have similar expected costs.

- Very high and very low estimates of hospital costs change the total expected costs of individual prostheses, but have little effect on their relative costs.

- In 70 year old women, a low price prosthesis is generally less costly than a high price prosthesis, even with a very low revision rate.

- In 40 year old women, prostheses with high prices and low revision rates can be less costly over 20 years than low priced prostheses with higher failure rates.

The results are intended to assist decision making, not to be a prescription for policy. The assumptions we made that may affect both the total expected costs and the relative costs of prostheses are as follows.

The cost of revision surgery compared with primary replacement in this model has been calculated from actual prices. Although this estimate seems low compared with some estimates, ${ }^{8}$ the sensitivity analysis showed that the extra costs of revisions were not a main driver of total expected costs. Uncertainty around the value of this variable is therefore not critical. However, we also assumed no re-revisions. Total expected costs would increase and relative costs may change if re-revisions were included in the model. For a prosthesis with a high revision rate, the cumulative effects of the costs of re-revision would be greater than for a prosthesis with a lower revision rate. If the extra costs of revisions were substantially greater than assumed, and potentially high re-revision rates were included in the model, total expected and relative costs may change. For example, high cost/low revision prostheses may become less costly than low cost/higher revision rate prostheses.

We assumed linear extrapolation of prosthesis survival data to 20 years. The survival rate of many prostheses is likely to fall (sometimes dramatically) over time. Increases in revision rates to levels above those assumed will increase total expected costs. Cost rankings will also be changed if rates of revision of prostheses change relative to each other.

We also assumed that people who have had a hip replacement have a mortality rate equal to the general population for their age group. If, as Malchau et $a l^{14}$ suggest, these people have on average a lower life expectancy, total expected costs would be lower than estimated here because of the greater number of people dying before their prosthesis needs replacing. It is possible, however, that younger patients may be a more active group than the general population and therefore have a higher life expectancy. More information is needed before the true impact on costs of life expectancy can be known.

Increases (decreases) in prosthesis costs used will increase (decrease) total expected costs. Changes in prosthesis prices relative to each other may also change relative costs. Costs at specific hospitals can be modelled to allow for differences in manufacturers' prices.

We did not calculate costs occurring after 20 years. For elderly patients, the discount factor combined with the high mortality rate means that expected costs in 20 years time are negligible $(£ 0.35$ in the 20th year after primary implant). However, for young patients, the low mortality rate and the increasing total hip replacement revision rate over time mean that a high proportion of younger people will survive longer than their implants. Despite the discount factor, the expected costs for a 40 year old recipient in the 20th year after primary implant are still relatively important ( $£ 26.48)$. This difference must be considered when comparing results across age groups.

Pynsent ${ }^{8}$ argues that a premium of $9 \%$ for 75-84 years olds $(20 \%$ for $65-74$ year olds $)$ should be charged for "lifetime" (20 year) insurance for the Charnley prosthesis. From our model, the premium for 70 year olds would be around $10 \%$ for the Charnley (expected revision costs of $£ 349$ for a primary replacement of $£ 3355$, table 1). For prostheses with 
higher expected rates of revision, the premium would be a proportionately higher percentage of primary replacement costs (16\% for MckeeFarrar and $22 \%$ for AML). We agree with Pynsent that a "lifetime" care package should encourage monitoring of prosthesis survival rates and setting of prices to reflect these rates. However, with hospital costs, prosthesis price, and revision rates as the components having the greatest effect on expected costs, such a proposal would be risky for providers (who would offer the package to purchasers). While providers have good knowledge of their own costs and the prices charged for prostheses, they would be required to set premiums based on information from manufacturers on prosthesis revision rates. Lack of published data and poor knowledge of long term revision rates may encourage reliance on established prostheses and thus stifle new developments. A solution would be to require all new prostheses to undergo long term clinical trials before release onto the market. EC directives state that, by June 1998, hip prostheses used in the UK (among other implants) should carry a "kite mark" showing Medical Devices Agency approval. ${ }^{18}$ This will not be as rigorous as clinical trials for drugs (which can take up to 10 years before being granted a licence), but is a first step. ${ }^{18}$

Despite data of variable quality and limited data on important characteristics, such as long term survival of prostheses, the approach outlined in this paper, based on total expected costs, assists decisions on choice of prostheses by making explicit those factors affecting total expected costs. The use of up to date hospital costs, combined with available (table 3 ) and potential (figures 1 and 2) revision rates, enables new prostheses to be assessed against those for which we have good data, and allows new assessments to be made relatively easily as data become available.

This paper has not investigated the effects on patients' quality of life of different prostheses, or of repeated and early revisions. An interesting area for future research would be to combine prosthesis survival and quality of life data to make these comparison.

We thank the staff at the two collaborating hospitals for supplying cost data and other information for use in this model. We also thank the authors of the systematic review of which this paper is a small part: Alex Faulkner, Gail Kennedy, Jenny Donovan, Mark Wilkinson, Ian Learmonth and Paul Gregg. Thank you also to two anonymous referees who supplied helpful comments on an earlier draft.

Funding: National Health Service Research \& Development Health Technology Assessment Programme of England and Wales.

Conflicts of interest: none.

1 Faulkner A, Kennedy LG, Baxter K, et al. Effectiveness of hip prostheses in primary total hip replacement: critical review of evidence and an economic model. Health Technol Assess 1998;2:1-133

2 Huiskes R. Failed innovation in total hip replacement: diagnosis and proposals for a cure. Acta Orthop Scand 1993;64 699-716.

3 Murray DW, Carr AJ, Bulstrode CJ. Which primary total hip replacement? F Bone foint Surg Br 1995;77:520-7.

4 Medical Devices Agency. Hazard notice. (MDA9801). London: MDA, 1998

5 Muirhead-Allwood SK. Lessons of a hip failure. BMF 1998; 316:644.

6 Daellenbach HG, Gillespie WJ, Crosbie P, et al. Economic appraisal of new technology in the absence of survival data - the case of total hip replacement. Soc Sci Med 1990;31:1287-93.

7 Gillespie WJ, Pekarsky B, O'Connell DL. Evaluation of new technologies for total hip replacement: economic modelling technologies for total hip replacement: economic modelling

and clinical trials. F Bone foint Surg Br 1995;77:528-33.
Pynsent PB, Carter SR, Bulstrode CJK. The total cost of hip-joint replacement: a model for purchasers. F Public Health Med 1996;18:157-68.

9 Briggs A, Sculpher M, Buxton M. Uncertainty in the economic evaluation of health care technologies: the role of sensitivity analysis. Health Econ 1994;3:95-104

10 Netten A, Dennett J. Unit costs of health and social care, 1997. Canterbury: University of Kent, Personal Social Services Research Unit, 1997.

11 Office of Population Censuses and Surveys. Mortality statistics. Series DH1, No. 27. London: HMSO, 1992.

12 August AC, Aldham CH, Pynsent PB. The McKee-Farrar hip arthroplasty: a long term study. $\mathcal{F}$ Bone Foint Surg $\mathrm{Br}$ 1986;68:520-7.

13 Ritter MA. The cemented acetabular component of a total hip replacement: all polyethylene versus metal backing. hip replacement: all polyethyl

14 Malchau H, Herberts P, Ahnfelt L. Prognosis of total hip Malchau H, Herberts P, Ahnfelt L. Prognosis of total hip
replacement in Sweden. Follow-up of 92,675 operations performed in 1978-1990. Acta Orthop Scand 1993;64:497506.

15 Nizard RS, Sedel L, Christel P, et al. Ten-year survivorship of cemented ceramic-ceramic total hip prosthesis. Clin Orthop 1992;282:53-63.

16 NHS Centre for Reviews and Dissemination. Total hip replacement. Effective Health Care 1996;2:1-12.

17 Krahn M, Gafni A. Discounting in the economic evaluation of health care interventions. Med Care 1993;31:403-18.

18 Bryan J. Keeping the ball rolling. Health Service fournal (Managers and Medicine) 1998;108:14-15. 